\title{
Confocal laser endomicroscopy in gastro-intestinal endoscopy: technical aspects and clinical applications
}

\author{
Nastazja Dagny Pilonis ${ }^{1,2,3}$, Wladyslaw Januszewicz ${ }^{2,3}$, Massimiliano di Pietro ${ }^{1}$ \\ ${ }^{1}$ MRC Cancer Unit at the University of Cambridge, Cambridge Biomedical Campus, Cambridge, UK; ${ }^{2}$ Department of Gastroenterology, \\ Hepatology and Clinical Oncology, Medical Centre for Postgraduate Education, Warsaw, Poland; ${ }^{3}$ Department of Gastroenterological Oncology, the \\ Maria Sklodowska-Curie Memorial Cancer Centre and Institute of Oncology, Warsaw, Poland \\ Contributions: (I) Conception and design: $M$ di Pietro; (II) Administrative support: None; (III) Provision of study materials or patients: None; (IV) \\ Collection and assembly of data: None; (V) Data analysis and interpretation: None; (VI) Manuscript writing: All authors; (VII) Final approval of \\ manuscript: All authors. \\ Correspondence to: Massimiliano di Pietro. MRC Cancer Unit at the University of Cambridge, Cambridge Biomedical Campus, Box197, CB20XZ, \\ Cambridge, UK. Email: md460@mrc-cu.cam.ac.uk.
}

\begin{abstract}
Confocal laser endomicroscopy (CLE) is an advanced endoscopic imaging technology that provides a magnified, cellular level view of gastrointestinal epithelia. In conjunction with topical or intravenous fluorescent dyes, CLE allows for an "optical biopsy" for real-time diagnosis. Two different CLE system have been used in clinical endoscopy, probe-based CLE (pCLE) and endoscope-based CLE (eCLE). Using pCLE, the device can be delivered: (I) into the luminal gastrointestinal tract through the working channel of standard endoscopes; (II) into extraluminal cystic and solid parenchymal lesions through an endoscopic ultrasound (EUS) needle; or (III) into the biliary system through an endoscopic retrograde cholangiopancreatography (ERCP) accessory channel. With eCLE, the probe is directly integrated into the tip of a conventional endoscope, however, these endoscopes are no longer commercially available. CLE has moderate to high diagnostic accuracy for neoplastic and inflammatory conditions through the gastrointestinal tract including: oesophageal, gastric and colonic neoplasia, pancreatic cysts and solid lesions, malignant pancreatobiliary strictures and inflammatory bowel disease. Some studies have demonstrated the diagnostic benefit of CLE imaging when combined with either conventional white light endoscopy or advanced imaging technologies. Therefore, optical biopsies using CLE can resolve diagnostic dilemmas in some cases where conventional imaging fails to achieve conclusive results. CLE could also reduce the requirement for extensive tissue sampling during surveillance procedures. In the future, CLE in combination with molecular probes, could allow for the molecular characterization of diseases and assess response to targeted therapy. However, the narrow field of view, high capital costs and specialized operator training requirements remain the main limitations. Future multi-center, randomized trials with a focus on conventional diagnostic applications, costeffectiveness and standardized training will be required for definitive evidence. The objective of this review is to evaluate the technical aspects and current applications of CLE in patients with gastrointestinal and pancreatobiliary diseases and discuss future directions for this technique.
\end{abstract}

Keywords: Confocal laser endomicroscopy (CLE); image enhanced endoscopy; esophageal cancer; stomach cancer; colon cancer; inflammatory bowel disease (IBD); pancreatic cancer; fluorescent endoscopy

Received: 08 September 2019; Accepted: 30 March 2020; Published: 25 January 2022.

doi: $10.21037 / \operatorname{tgh} .2020 .04 .02$

View this article at: http://dx.doi.org/10.21037/tgh.2020.04.02 


\section{Technical aspects}

\section{Introduction}

Confocal laser endomicroscopy (CLE) and endocytoscopy are the two endoscopic technologies that provide diagnostic information at cellular level in real-time. Endocytoscopy will be discussed in a separate chapter. CLE develops on the background of confocal microscopy, which was introduced in 1957 by Marvin Minsky, to address the practical issue of imaging tissue at different depths below the surface. As such, CLE allows the endoscopist to image gastro-intestinal (GI) epithelia underneath what is conventionally seen with standard and image enhanced endoscopy. The term confocal refers to the property of align illumination and collection system in the same specimen plane. To this end, a point light source is generated by converging the beam from a low power laser (488 $\mathrm{nm}$ blue laser light) on a single point. This is achieved using a source pinhole, the reflection of the light by a beam splitter and an objective lens to focus the light (Figure 1). The light hitting the specimen will converge onto a fixed plane and generate fluorescence. The fluorescence emitted from the specimen will be collected by the objective lens and delivered to the detector. However, a detector pinhole will reject signal coming from the outof-focus areas; in this way, only the light from the focal point can be collected (Figure 1). Two different CLE system have been used in clinical endoscopy, the probebased CLE (pCLE) and the endoscope-based CLE (eCLE), of which the latter is no longer commercially available. To allow tissue excitation CLE systems require the use of fluorescence dyes, which can be topical or intravenous (IV). Topic dyes include cresyl violet and acriflavine. Cresyl violet enhances the cytoplasm but has limited tissue penetration and does not provide an information about vasculature; cresyl violet has very limited evidence in GI endoscopy (1). Acriflavin stains the nuclei of the surface epithelial cells, but has limited penetration into the deeper layer (2). The concentration used varies between 0.02 and $0.05 \%$, however some concerns about mutagenic and cytotoxic properties have limited the use in clinical practice $(3,4)$. Fluorescein is the most common intravenous dye used in CLE and it is FDA-cleared. Fluorescein is safe and routinely used in ophthalmology for diagnostic angiography of the retina. In CLE, $2.5 \mathrm{~mL}$ of $10 \%$ solution is typically administered immediately before endomicroscopic examination and the fluorescence is optimally captured from few seconds to 8 minutes after, but as long as 60 minutes after injection. In a survey of 16 centres performing fluorescein-aided
CLE mild adverse events occurred in $1.4 \%$ of cases and included nausea, vomiting, mild epigastric pain, transient hypotension (without shock), rash at the site of injection and diffuse erythema (5).

\section{$p C L E$}

The pCLE (Mauna Kea Technologies; Paris, France) is a fibre bundle that transits into the GI tract through the working channel of a standard endoscope (Figure 2). A variation of this is the needle-based CLE (nCLE), which can be delivered by through an endoscopic ultrasound (EUS) needle in cystic (e.g., pancreas) or solid extraluminal lesions. In pCLE, the individual fibres act as pinhole and the optical lens is integrated in the tip of the probe. pCLE has a fixed plane of imaging. The pCLE is available as 4 different GI probes, GastroFlex, ConolFlex, CholangioFlex and AQFlex 19, which scan with a frame rate ranging between 9 and 12 images per second. The GastroFlex and ColoFlex are compatible with standard endoscope with $2.8 \mathrm{~mm}$ working channel, have a depth of focus between 55 and $65 \mu \mathrm{m}$, a field of view of $240 \mu \mathrm{m}$ and a lateral resolution of $1 \mu \mathrm{m}$. The CholangioFlex is passed through an endoscopic retrograde cholangiopancreatography (ERCP) accessory channel of at least $1 \mathrm{~mm}$, has a depth of focus between 40 and $70 \mu \mathrm{m}$, a field of view of $325 \mu \mathrm{m}$ and a lateral resolution of $3.5 \mu \mathrm{m}$. The AQ-Flex 19 nCLE has the same depth of focus, field of view and lateral resolution of the CholangioFlex, but is advanced through a 19G EUS fine-needle aspiration (FNA) needle. These pCLEs are reusable (maximum number of examination of 20 GastroFlex and ColoFlex and 10 for CholangioFlex and AQ-Flex 19) and can be cleaned by most of commercially available endoscopy reprocessing machines. The capital investment to acquire the system consists of an endoscopy stack (Figure 2), which typically include the light source, the image processor, a keyboard, a monitor, a pedal switch to activate the light and control the image/video recording and a track ball to back/forward view live images.

\section{$e C L E$}

In eCLE confocal microscope is fitted at the distal end of an endoscope (Pentax, Tokyo) (Figure 2). Having a single machine for conventional endoscopy of upper and lower GI tract and CLE of targeted lesions is an advantage. However, the endoscope has the downside of large diameter $(12.8 \mathrm{~mm})$ and a $5 \mathrm{~cm}$ rigid distal end, which particularly in upper GI, can make intubation less straightforward. The 


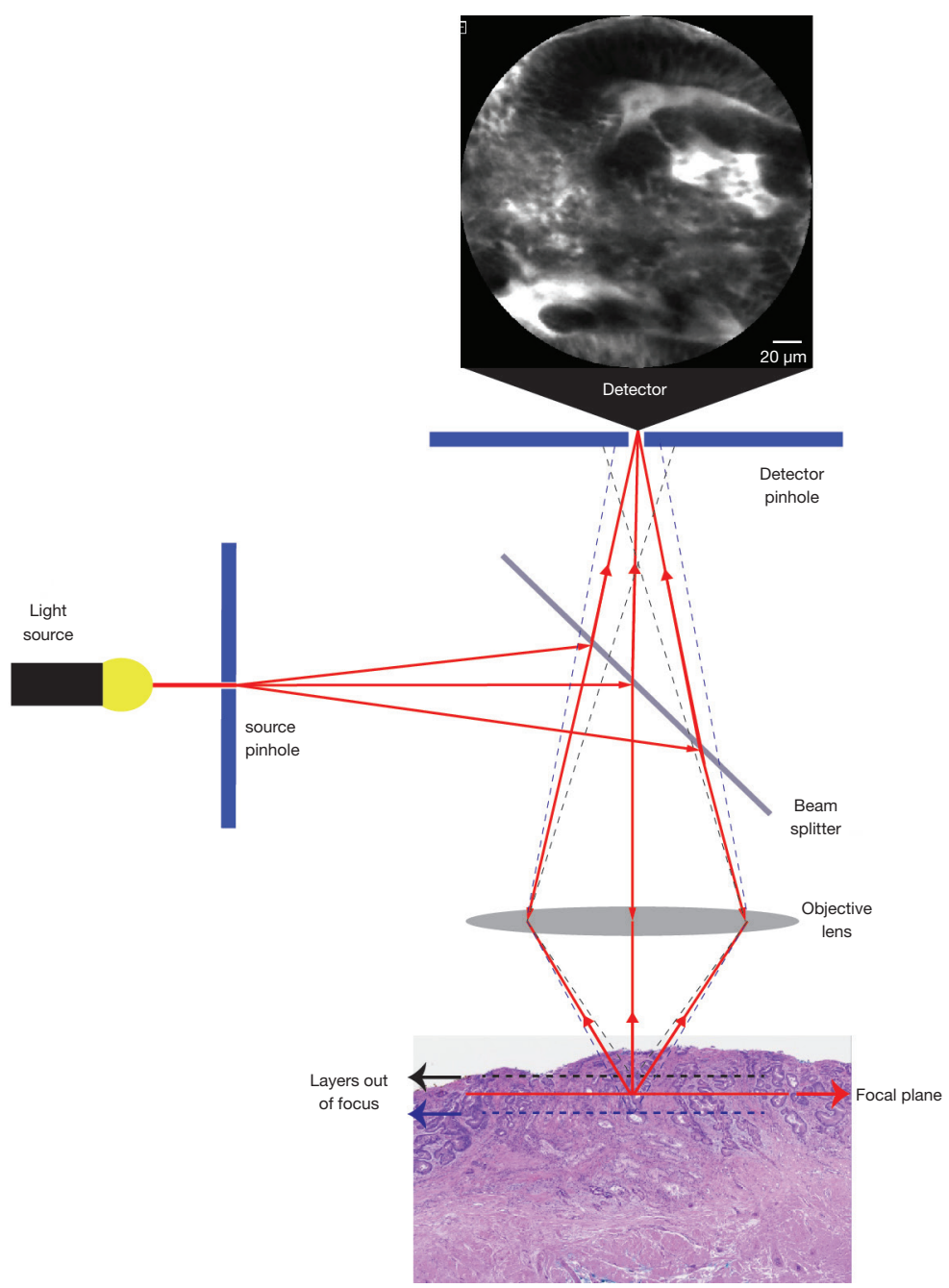

Figure 1 Principles of confocal laser endomicroscopy. The light passing through a pinhole is reflected by the beam splitter and then focused by the objective lens. The tissue is illuminated and the fluorescence emitted is collected by the objective. A pin hole placed before the detector allows to reject the light signal from out-of-focus tissue layers. Magnification: 10x.

eCLE produces videos with a significantly lower frame rate (1.6/s), but allows to adjust the depth of scanning between 0 and $250 \mu \mathrm{m}$. It also has a better resolution then pCLE (0.7 $\mu \mathrm{m}$ lateral and $7 \mu \mathrm{m}$ axial). Unfortunately, this system is no longer commercially available.

\section{Considerations on training}

The available evidence suggests that interpretation of CLE patterns is easy and subject to a steep learning curve by assessors with no previous experience in the technology and across different GI diseases. Although online material is available for training in pCLE (www.cellvizio.net), one randomised study comparing in-class versus selfdirected training showed that a formal teaching session by experienced trainer was more effective than self-directed training (6). The majority of the studies indicate that after a formal teaching session endoscopists maintain stable levels of diagnostic accuracy when assessing blocks of cases of variable size to an overall sample size variable between 76 and 90 (7-9). One study indicates that the diagnostic accuracy for colorectal neoplastic lesions by 11 nonexperienced endoscopists after formal training session improved from $63 \%$ in the first block of 20 videos to $86 \%$ in the fourth block of clips (10). Overall, it is recommended that after a formal training, self-directed skills consolidation 

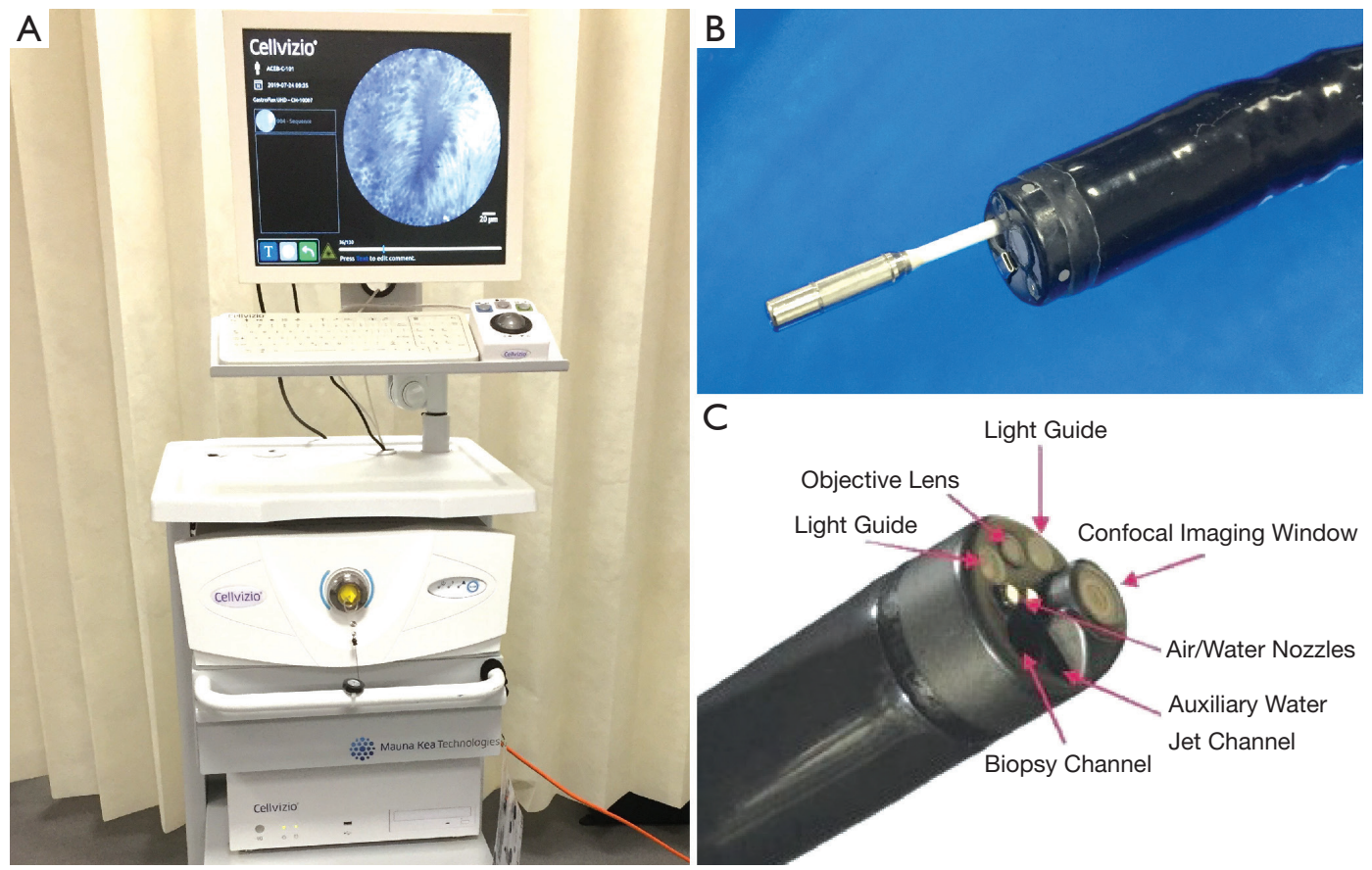

Figure 2 CLE technologies. (A) Probe-based confocal laser endomicroscopy system (pCLE) (Mauna Kea Technologies, Paris, France). (B) pCLE probe can be delivered in vivo through the standard $2.8 \mathrm{~mm}$ endoscope channel. (C) Endoscope-based confocal laser endomicroscopy (eCLE). The arrows point to the different components of the eCLE system. Adapted from De Palma GD, World 7 Gastroenterol, 2009. CLE, confocal laser endomicroscopy.

through assessment of 50-80 cases be undertaken to achieve diagnostic proficiency. However, ability to interpret CLE images does not translate in ability to generate good quality images and to make diagnosis during a live procedure. There is limited evidence on the learning curve to perform CLE and make diagnosis in real-time.

\section{Clinical applications}

\section{Oesophagus}

Several studies have provided evidence on the clinical utility of CLE for diagnosis of oesophageal premalignant lesions and neoplasms. The majority of these studies have focused on oesophageal adenocarcinoma (OAC) and Barrett's oesophagus (BE) with low-grade dysplasia (LGD) and highgrade dysplasia (HGD). The current recommendation for $\mathrm{BE}$ surveillance is to take targeted biopsies and random biopsies according to the Seattle protocol $(11,12)$. Since dysplastic changes are patchy and can be completely invisible the diagnostic accuracy of the Seattle protocol is limited by sampling error and high costs related to multiple biopsies. In this context CLE offers the potential advantage help direct biopsies and possibly increase the diagnostic accuracy, as well as reduce the number of random biopsies.

The non-dysplastic BE epithelium is easily recognizable in confocal imaging (Figure 3). CLE displays the mucin contained in goblet cells as characteristic dark roundappearing structures within the columnar cells, which are easily recognizable by the bright contours (13). The glands have linear contours and the interglandular space is regular in size. The pCLE criteria for the diagnosis of HGD and intramucosal adenocarcinoma have been defined in a study by Gaddam et al. (9). Six criteria with the highest accuracy $(81.5 \%)$ in predicting BE neoplasia were selected, including (I) "saw-toothed" epithelial surface; (II) poorly identifiable goblet cells; (III) non-equidistant glands; (IV) glands unequal in size and shape; (V) enlarged cells; (VI) non-equidistant and irregular cells (Figure 4). Two positive criteria provided the best accuracy for a diagnosis of BE-related neoplasia. A substantial interobserver agreement was achieved $[\kappa=0.61(0.53-0.69)]$. By including only highquality videos, accuracy and agreement rose to $95 \%$ and $\kappa=0.89$, respectively. 


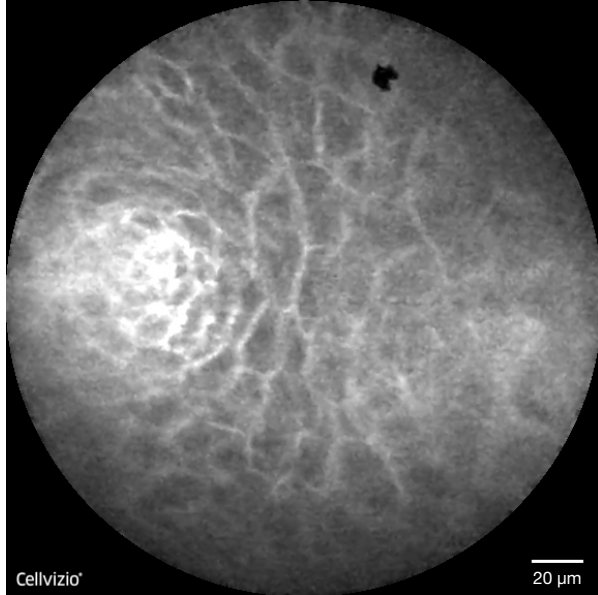

Figure 3 Normal squamous epithelium in oesophagus-dark, homogeneous epithelial cells with regular architecture and clearly visible borders, capillaries directed toward the epithelium within the lamina propria, without penetration of the contrast agent into the surrounding tissue. Adapted from Templeton A, et al., Clin Endosc, 2013.

Recently, pCLE criteria for LGD have been developed (14). The CLE features of LGD are similar to those of more advanced dysplasia however reflect the more subtle histological abnormalities. pCLE criteria for LGD include (I) dark non-round glands; (II) irregular gland shape; (III) lack of goblet cells; (IV) variable degree of darkness with sharp cut-off; (V) variable cell size; and (VI) cellular stratification (Figure 4). A cut-off of 3 out of 6 positive criteria yielded a sensitivity and specificity of $81.6 \%$ and $67.6 \%$, respectively, with an area under the ROC (AROC) of 0.860 for LGD diagnosis. The overall agreement among six endoscopists was good, with a $\kappa$ value of 0.631 , with no difference in the diagnostic accuracy between experienced and non-experienced endoscopists. Two randomised studies have been performed investigating CLE for Barrett's early neoplasia diagnosis (HGD/intramucosal cancer). One study randomised 101 patients to either WLE or NBI first followed by pCLE on targeted and random locations. pCLE was therefore not part of the randomisation process. In this study the combination of pCLE and WLE achieved 93\% sensitivity and $67 \%$ specificity, with 41 additional neoplastic locations found by pCLE compared to WLE alone (15). In another study, 192 patients were randomised to receive either WLE with Seattle protocol or WLE with eCLE and targeted biopsies only. In this study, eCLE increased the sensitivity for neoplasia detection from $40 \%$ to $96 \%$
$(\mathrm{P}<0.001)$, with $92 \%$ specificity $(16)$.

The overall accuracy of CLE in diagnosing BE-related neoplasia was recently reviewed in a meta-analysis including eight studies (both e-CLE and p-CLE devices) from 709 patients (17). The pooled sensitivity and specificity for BE-related neoplasia diagnosis was 89\% [95\% confidence interval (CI), 0.80-0.95] and 75\% (95\% CI, 0.69-0.81), respectively, with an AUC of 0.947 . In a per-location analysis, the pooled sensitivity dropped to $70 \%$ (95\% CI, 0.65-0.74), and the specificity was $91 \%$ (95\% CI, $0.90-0.92$ ) with an area under the curve (AUC) of 0.951. When compared, the diagnostic performance of eCLE and pCLE did not differ significantly. Of note, the sampling error is a serious limitation influencing the sensitivity of CLE-related studies in a per-location analysis as the biopsy sites may not always correspond to the area assessed by CLE. One solution to the narrow field of view when evaluating inconspicuous mucosa is the combination of CLE with endoscopic flagging techniques. For example, autofluorescence imaging (AFI) has been used in BE to guide pCLE to areas of potential dysplasia (18). In a singlecentre study on 55 patients with BE, the accuracy of AFItargeted pCLE for dysplasia was good with overall sensitivity and specificity of $96.4 \%$ and $74.1 \%$. The time required to perform AFI-targeted pCLE was significantly shorter than that taken by the Seattle protocol $(\mathrm{P}<0.001)(18)$.

The role of CLE in the diagnosis of early oesophageal squamous cell cancer (OSCC) has been investigated in a limited number of studies. The CLE features of normal squamous epithelium included dark, homogeneous epithelial cells with a regular architecture and clearly visible borders and capillaries, without penetration of the contrast agent into the surrounding tissue (Figure 3). The neoplastic tissue is characterized by dark cells with an irregular architecture, varying sizes, and lack of clearly visible borders, with signs of neo-angiogenesis (twisted, irregular, and elongated tumor vessels with a larger diameter) and leakage of fluorescein through the capillary walls (Figure 5) (19). In a small prospective cohort study on 21 patients with suspected early OSCC confocal imaging with eCLE was performed on a total 43 unstained areas after $0.5 \%$ Lugol's solution chromoendoscopy (19). Two endoscopists, blinded to the histology and endoscopic appearance of the lesions, reviewed the confocal images. The overall accuracy in providing OSCC diagnosis was $95 \%$, and the sensitivity and specificity were $100 \%$ and $87 \%$, respectively. Intraobserver agreement was almost perfect $(\kappa=0.95)$, and interobserver agreement 

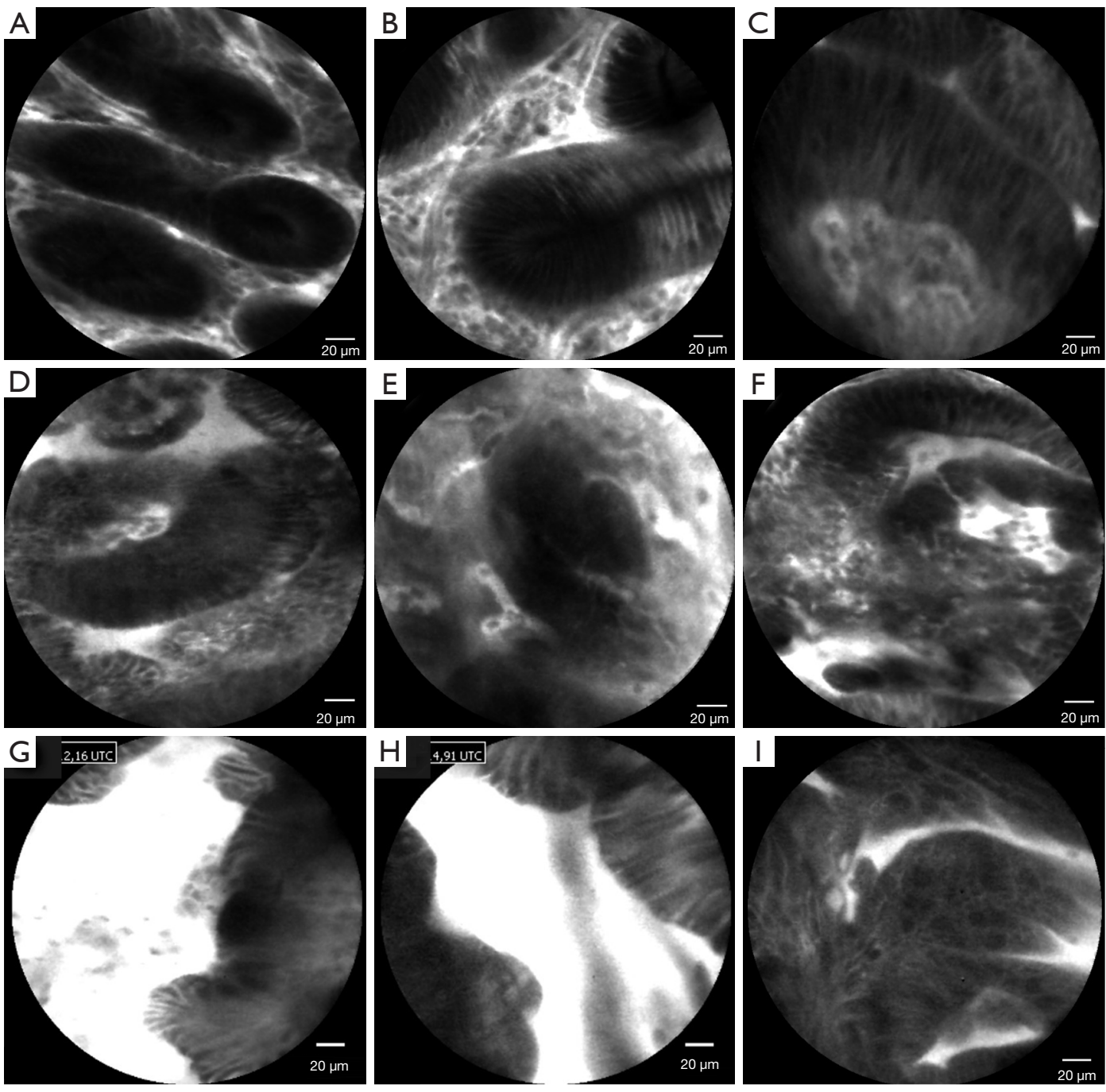

LGD 3/6

HGD 2/6

Figure 4 pCLE criteria for low-grade dysplasia and high-grade dysplasia in Barrett's oesophagus. (A) Dark non-round glands. (B) Variable degree of darkness with sharp cut-off. (C) Cellular stratification. (D) Poorly identifiable goblet cells. (E) Glands unequal in size and shape. (F) Non-equidistant and irregular cells. (G) "Saw-toothed" epithelial surface. (H) Non-equidistant glands. (I) Enlarged cells. pCLE, probebased confocal laser endomicroscopy.

was substantial ( $\kappa=0.79)$. A more recent study analysed the utility of pCLE in a cohort of patients with previous head and neck cancer and Lugol-unstained lesions (20). pCLE could provide correct diagnosis in 34 of the 37 unstained areas. The sensitivity, specificity, and accuracy of pCLE for the histologic diagnosis of OSCC in this cohort of patients were $94.1 \%, 90 \%$, and $91.9 \%$, respectively.

\section{Stomach}

CLE has been widely applied for the detection of neoplastic lesions and premalignant conditions of gastric cancer (GC), such as atrophic gastritis (AG) or intestinal metaplasia (IM).

The first classification of CLE images describing seven types of gastric pit patterns correlating with the disease spectrum ranging from normal mucosa, through AG /IM, up to early GC (Miami classification) was developed in 2008 (21). Recently, this classification was refined to include the vessel architecture along with the gastric pit patterns (22). Type I gastric pit pattern (regular pits with round/wide/ slit-like openings) corresponding to normal mucosa (in cardia/corpus/antrum, respectively); type II was subdivided 


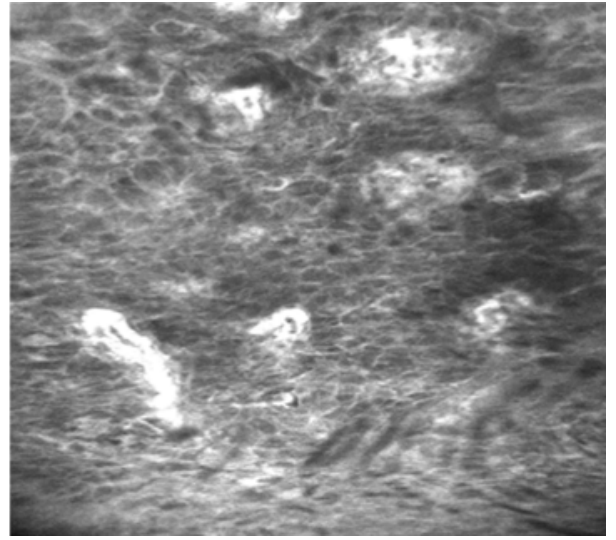

Figure 5 Oesophageal squamous carcinoma-squamous cells are irregularly arranged and differ in size and morphology. Capillary leakage of fluorescein is observed. Magnification: $1,000 \times$. Adapted from Liu J, et al., PLoS One, 2014.

into IIa (regular pits with elongated openings, increased fluorescence in stroma) which represented inflammatory mucosa; IIb (reduced pits with dilated openings) corresponding to atrophic mucosa, and type IIc (appearance of goblet cells with dark mucin), which represents IM; type III was subdivided into IIIa (mild to moderate irregular pits with variable width of the epithelial lining) which represents low-grade intraepithelial neoplasia; type IIIb (prominent distorted pits with irregular epithelial lining), corresponding to high-grade intraepithelial neoplasia and type IIIc (atypical glands/dispersion of irregular dark cells) diagnostic for differentiated/poorly differentiated adenocarcinoma (22). Vessel architecture was also classified into 3 types. Normal mucosa showed type I (regular capillaries with normal calibre, anfractous/honeycomb-like/coil-shaped for cardia/corpus/antrum, respectively), inflammatory gastric mucosa showed type II (increased capillaries with elevated leakage), while neoplastic gastric mucosa mainly showed type III (irregular capillaries with heterogenous leakage/ dilated calibre) (22). The sensitivity and specificity of the established pCLE criteria were $89 \%$ and $99 \%$, respectively, for $\mathrm{AG}, 92 \%$ and $99 \%$, respectively for $\mathrm{IM}$, and $90 \%$ and $99 \%$, respectively, for GC. The interobserver agreement for the differentiation of neoplastic versus non-neoplastic lesions was substantial (kappa=0.70) (22). The pCLE appearance normal and pathological gastric mucosa are represented in Figure 6.

Two meta-analyses showed a high diagnostic value of eCLE in the detection of AG, IM and GC. The sensitivity and specificity for the diagnosis of AG were $88 \%$ and $98 \%$, respectively, and 93\% and 98\%, respectively, for IM (23). Pooled sensitivity and specificity for gastric neoplasia were $81 \%$ and $98 \%$, respectively. High sensitivity and specificity have also been demonstrated for the diagnosis of gastric adenocarcinoma (89\% and 99\%, respectively) (24). The eCLE appearance normal and pathological gastric mucosa are represented in Figure 7.

A double-blind study randomized 168 patients to undergo standard white-light endoscopy (WLE) and eCLE with targeted biopsies or WLE with a standard random biopsy protocol and showed no difference in diagnostic accuracy in a per-patient analysis. However, a per-biopsy analysis revealed that targeted biopsies with CLE had a significantly higher yield for IM than WLE (66\% vs. 16\%, respectively). The number of targeted biopsies needed to confirm IM was about one-third of that needed for WLE with standard biopsies (25). These data suggest that CLE can help reduce the number of biopsies for a diagnosis of gastric IM. However, given the large surface gastric area and the patchy distribution of gastric IM/dysplasia the narrow field of view of CLE represents a limitation, warranting a combination with broad-field (red-flag) techniques. A study comparing the diagnostic yield of magnifying flexible spectral imaging color enhancement (ME-FICE) alone and ME-FICE plus pCLE for IM detection, showed that ME-FICE followed by pCLE provided higher specificity ( $90 \%$ vs. $79 \%$ ) and positive predictive value (PPV; $86 \%$ vs. 74\%), however, similar sensitivity and negative predictive values (NPV; $96 \%$ and $97 \%$, respectively) as compared to ME-FICE alone (26).

Those results were further supported by the evidence from a randomised controlled trial (RCT), which revealed that real-time pCLE targeted biopsies after FICE significantly improved the diagnostic yield of IM and early gastric cancer and reduced the number of biopsies required as compared to FICE with standard biopsies $(75.1 \%$ vs. $31.5 \%$ on a per-biopsy analysis). However, the per-patient analysis showed no difference between the groups (27).

Similar results were obtained in combination with narrow-band imaging (NBI) (28).

Lastly, CLE was showed to enable to visualization of $H$. pylori in combination with acriflavine staining (29) and lead to high sensitivity and specificity $(93 \%$ and $86 \%$, respectively) for a diagnosis of $H$. pylori-related gastritis yielded (30). However, the oncogenic potential of acriflavine remains an important limitation of the method. 

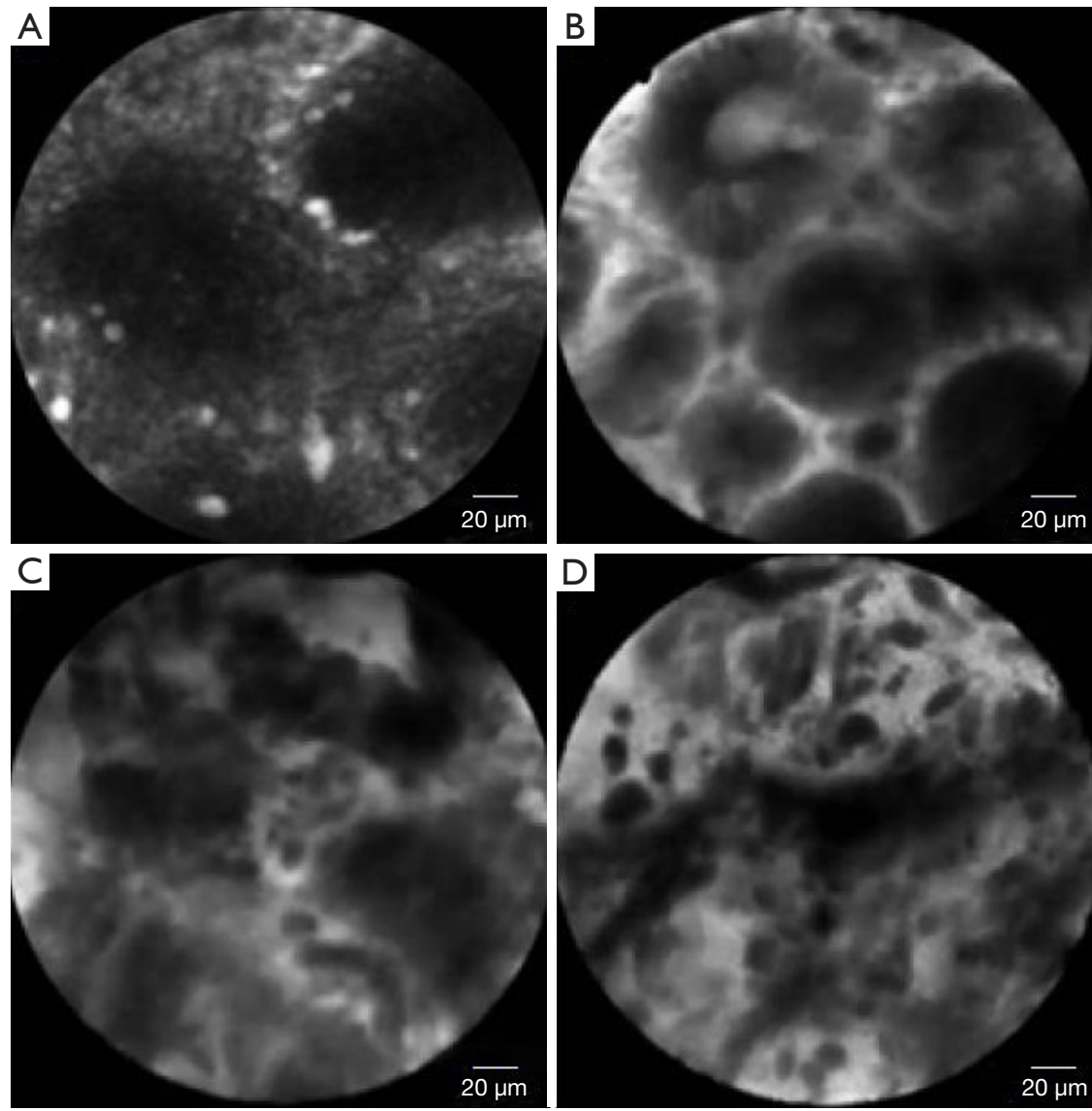

Figure 6 The pCLE appearance of normal and pathological gastric mucosa. (A) Normal cardiac mucosa-regular pits with wide openings. (B) Dysplastic gastric-dark epithelium with irregular and varying thickness is observed. (C) Differentiated adenocarcinoma-disorganized epithelium with dark and irregular glands. (D) Undifferentiated adenocarcinoma-dark and irregular cells with no identifiable glandular structures are observed. Adapted from Kin MY, et al., World 7 Gastroenterol, 2016. pCLE, probe-based confocal laser endomicroscopy.

\section{Small bowel}

The usefulness of CLE in the diagnosis of small bowel pathologies has been investigated only in a small number of studies. A study of 38 pCLE examinations performed during a double-balloon enteroscopy, identified characteristic pCLE features of various small-bowel diseases based on the size of the capillary calibres and lymphatic vessels. CLE pathological features correctly correlated with histopathologic diagnosis in $91 \%$ cases (31).

The endomicroscopic criteria for celiac disease (CD) include villous atrophy (presence of $\geq 5$ blunt-shaped villi observed on superficial scans), crypt hyperplasia (presence of $\geq 1$ crypt on small bowel deep CLE imaging) (Figure 8, and increased number of intraepithelial lymphocytes (IELs $>40 / 100$ enterocytes) (32-34). A meta-analysis performed on three studies with 110 patients showed a $84 \%$ sensitivity and $94 \%$ specificity for CLE in the diagnosis of CD (30).

One single-center study comparing dual-focus NBI and pCLE for real-time diagnosis of adenomatous polyps in patients with FAP, showed that pCLE had a similar, high degree of diagnostic value as compared with NBI (35).

Interestingly, confocal endomicroscopy can visualise food-associated changes in the duodenal mucosa of patients with irritable bowel syndrome (IBS). CLE showed a realtime response to food antigens in 22 of 36 patients with IBS and previously undetected allergies exposed to the diluted food antigens directly applied endoscopically to the duodenal mucosa compared to control sterile water with simethicone. As early as 5 minutes, CLE detected increased IELs, epithelial leaks, and intervillous space widening. The most reproducible phenomenon was the eruption 

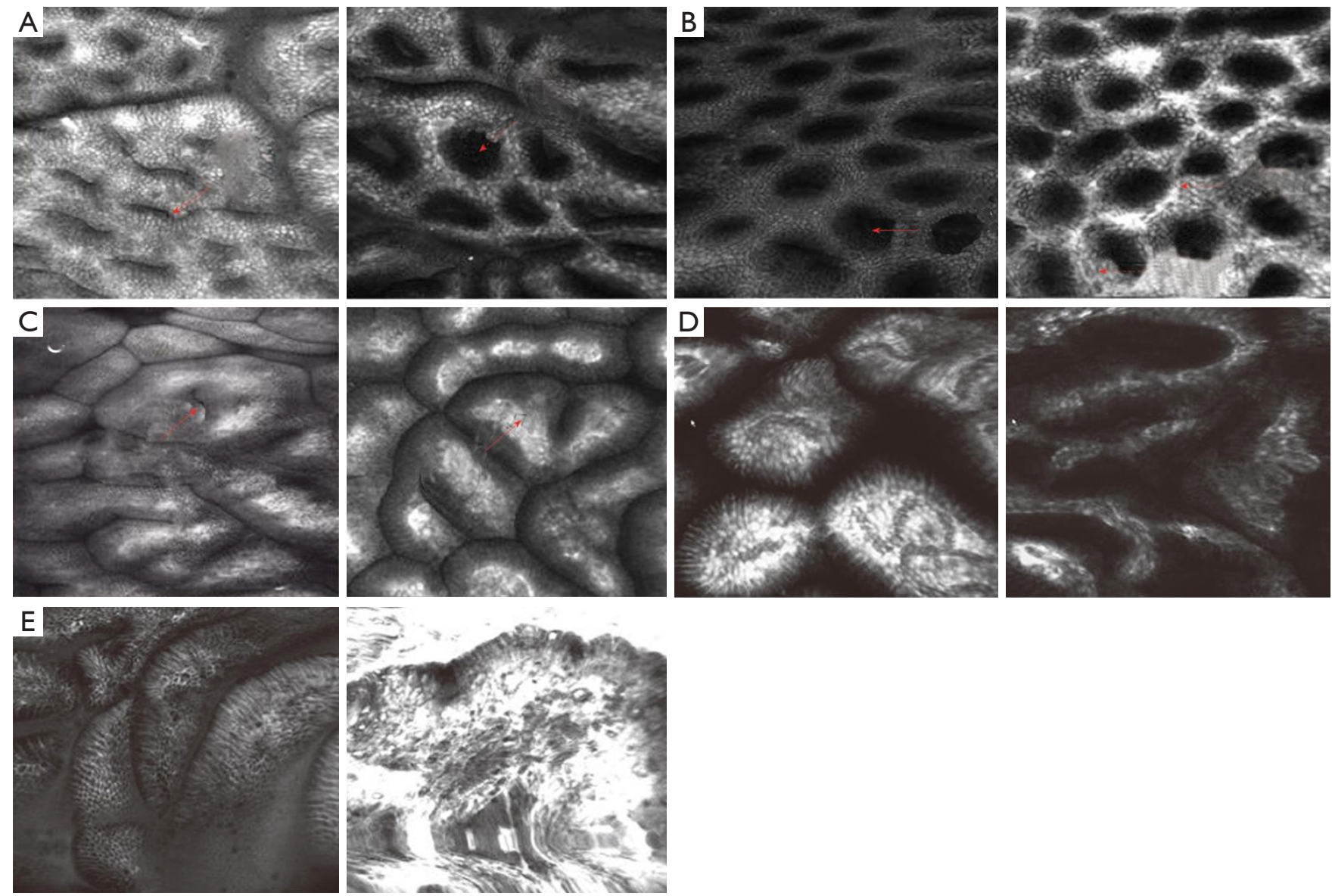

Figure 7 The eCLE appearance of normal and pathological gastric mucosa. (A) Normal gastric mucosa in the fundus - gastric pits are round (solid arrow), net-like subepithelial capillary network patterns surround the gastric pits (dash arrow). (B) Normal gastric mucosa in the body-gastric pits are round (solid arrow). Honeycomb-like subepithelial capillary network patterns surround the gastric pits (dash arrows). (C) Normal gastric mucosa in the antrum-gastric pits are the line type (solid arrow). Coil-shaped subepithelial capillary network patterns surround the gastric pits (dash arrow). (D) Low grade gastric intraepithelial neoplasia-different sizes of gastric pits, capillary network is thickening and circuitous. (E) High grade gastric intraepithelial neoplasia-abnormal arrangement of gastric pits. The thickening capillary network and the increasing branch present a mass shape. Magnification: 1,000x. Adapted from Shu-Fang W, et al., World $\mathcal{7}$ Gastroenterol, 2012. eCLE, endoscope-based confocal laser endomicroscopy.

of fluorescein from the newly created leaks into the gut lumen. In this study IBS patients placed on exclusion diets informed by the CLE findings underwent improvement of their symptom scores by $74 \%$ at 12 months of followup (36). However, CLE remains an invasive test and the current protocol requires long intubation time, which can be rather impractical as routine diagnostic modality.

\section{Pancreas}

Most of the studies on the use of CLE in pancreatic pathology refers to the characterization of pancreatic cystic lesions (PCLs), with a small number of studies that more recently evaluated CLE in the diagnosis of solid lesions.

Several studies established the safety and feasibility of needle-based confocal laser endomicroscopy (nCLE) for PCLs with an overall diagnostic accuracy ranging between $46 \%$ and $95 \%$ (37-42). Many of these studies, however, are affected by the lack of a suitable gold standard in many cases, where the combination of EUS, cytology and fluid molecular analysis may not provide a definitive diagnosis. In such cases, conclusive histopathology on surgical specimens is not always achieved. The diagnostic criteria for PCLs on nCLE imaging were proposed on the basis of 

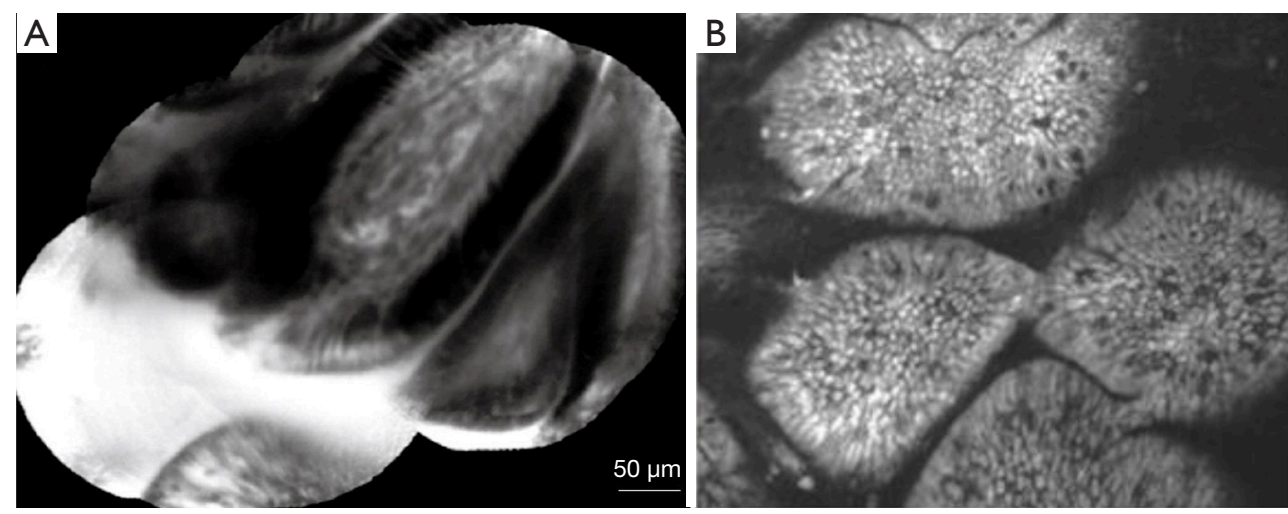

Figure 8 The CLE appearance of normal and pathological small bowel mucosa. (A) Normal small bowel mucosa-normal epithelium border with regular capillary pattern. (B) Confocal image of celiac disease-Marsh type 3b. Magnification: 1,000×. Adapted from De Palma, GD, World 7 Gastroenterol, 2009; Venkatesh K, World 7 Gastroenterol, 2009. CLE, confocal laser endomicroscopy.
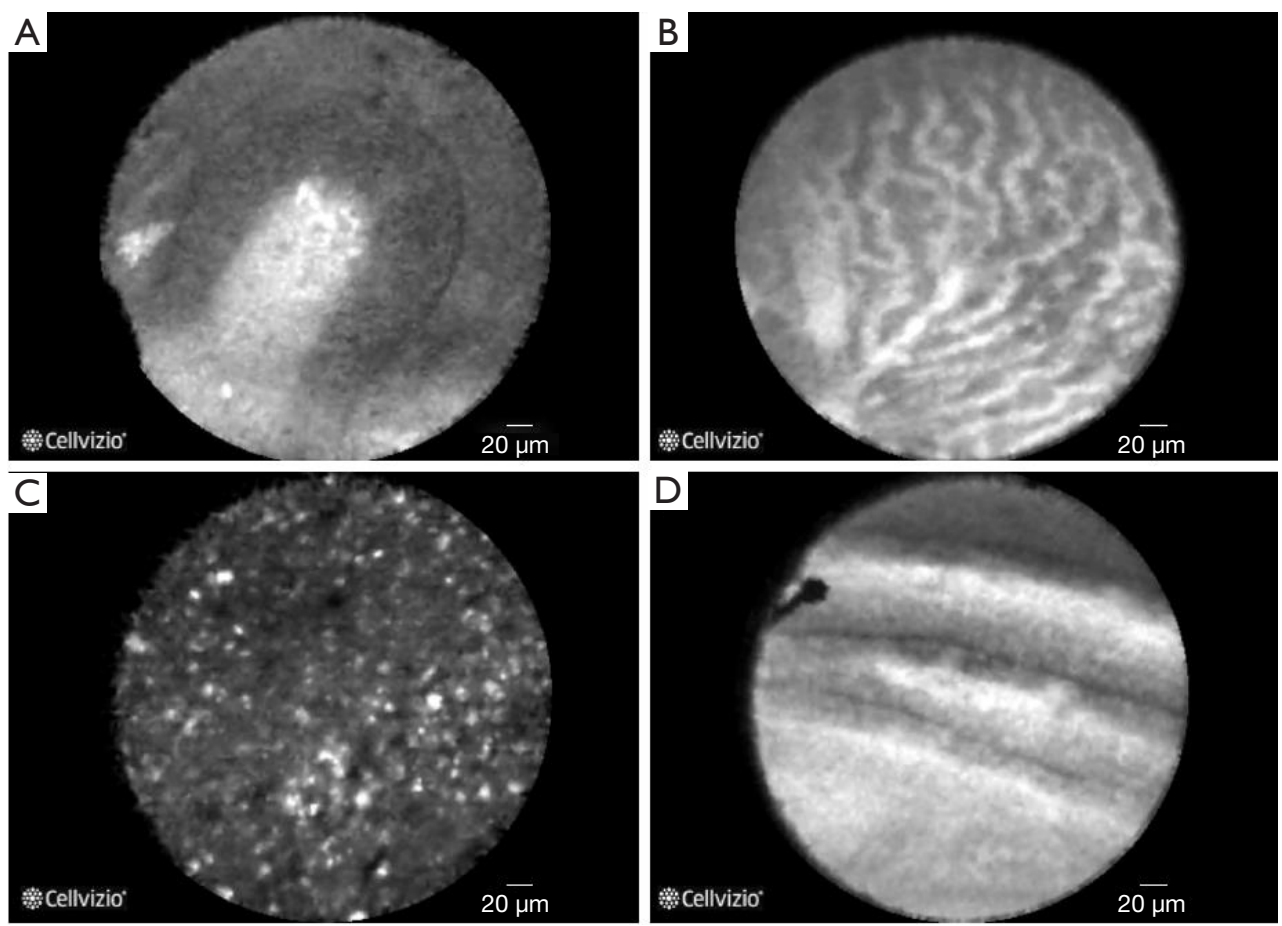

Figure 9 nCLE images of pancreas. (A) Intraductal papillary mucinous neoplasm: a single papilla with a central fibrovascular core and overlying epithelium. (B) Serous cystadenoma: branching and tortuous network of multiple blood vessels in a "fern like" pattern. (C) Pseudocyst: clusters of bright, floating particles with a background which is nondescript and lacks blood vessels. (D) Mucinous cystic neoplasm: solitary epithelial bands without formation of papillae. Adapted from Krishna G, et al., World f Gastroenterol, 2015. nCLE, needlebased confocal laser endomicroscopy.

three studies $(39,42,43)$. The specificity for the diagnosis of intraductal papillary mucinous neoplasms (IPMNs) and serous cystadenomas (SCAs) neared 100\% when fingerlike papillae and superficial vascular network pattern were visualised, respectively (Figure 9) $(38,40)$. However, in a study with 66 patients, the characteristic features for PCLs were associated with a modest sensitivity of $59 \%$ and a NPV of $50 \%$ (44). In a subsequent study the 
predefined typical structures for mucinous cysts were also associated with a modest sensitivity of $66 \%$ (45). The low sensitivity of nCLE may be increased by combining the method with cystoscopy. A study of 18 patients evaluated the combination of nCLE and cystoscopy in the diagnosis of PCLs, focusing on two specific features-mucin on cystoscopy and papillary projections with dark rings on nCLE. While the sensitivity of cystoscopy alone was $90 \%$, and that of nCLE was $80 \%$, the two methods combined had the sensitivity of $100 \%$ (40). A recent multi-center study of 78 PCLs demonstrated the superiority of CLE over EUS and carcinoembryonic antigen (CEA) for the diagnosis of large (mean size of $40 \mathrm{~mm}$ ), single, noncommunicating PCLs. In this study, the sensitivity and specificity of nCLE for the diagnosis of SCA, mucinous cystadenoma, branch duct IPMNs, cystic neuroendocrine neoplasm, cystic solid pseudopapillary neoplasm, and cystic lymphomas were all $\geq 95 \%$ (42). However, in clinical practice the differential diagnosis of PCLs is often achieved by cyst fluid analysis, cross-sectional imaging and clinical history. Therefore, the definitive evidence that $\mathrm{nCLE}$ can improve characterization of PCLs is awaited.

The studies on nCLE diagnoses of solid pancreatic lesions are limited. Giovannini et al. described nCLE criteria for pancreatic adenocarcinoma and neuroendocrine tumor (NET) (46). These diagnostic criteria were validated on a small of a cohort of 32 patients referred for EUS-FNA of solid pancreatic lesions. nCLE achieved sensitivities and specificities of $77 \%$ and $100 \%$, respectively, for a diagnosis of adenocarcinoma, $100 \%$ and $97 \%$, respectively, for NET and $50 \%$ and $100 \%$, respectively, for chronic pancreatitis (46). Another prospective pilot study on 22 patients with solid pancreatic lesions revealed accuracy for nCLE of 90.9\% (47). This level of diagnostic accuracy was not confirmed in a recent prospective dual-center study on 28 patients with pancreatic masses suspicious for malignancy, who underwent nCLE imaging. In this study, nCLE criteria showed sensitivity and specificity ranging between 19$93 \%$ and $0-56 \%$, respectively. Moreover, the interobserver agreement was very poor (48). Overall, the studies on pancreatic pathologies are limited by small number of patients and restricted to few expert centres. There are no data on the learning curve, so it is difficult to conclude whether skills on generation and interpretation of images can be easily acquired by endoscopists.

\section{Biliary strictures}

Conventional diagnosis of indeterminate bile duct strictures remains challenging due to the low sensitivity of ERCPguided biopsy sampling or brush cytology ( $48 \%$ and $45 \%$, respectively) $(49,50)$. The feasibility of intubating the biliary tree with the CholangioFlex probe and performing real time imaging has been shown to be high in two large studies that enrolled 222 patients, where pCLE was technically successful in $96 \%(51,52)$ with excellent risk profile (51-53). To date, two classification systems have been established to allow differential diagnosis in biliary strictures. The Miami Criteria include the following five features: white bands $>20 \mu \mathrm{m}$, dark bands $>40 \mu \mathrm{m}$, dark clumps, epithelial structures, or fluorescein leakage (Figure 10B-10E). The presence of two out of five criteria has sensitivity and specificity for the diagnosis of malignancy of $97 \%$ and $33 \%$, respectively (54). The low specificity associated with the Miami classification has been attributed to inflammatory changes. For more accurate differentiation between malignant and inflammatory changes, four additional inflammation-specific criteria have been developed (Paris classification) including vascular congestion, granular pattern with scales, increased inter-glandular space and thickened reticular structures (Figure 10F-10I) (55). A simultaneous evaluation of indeterminate pancreaticobiliary strictures with both the Miami and Paris classifications has been shown to increase the specificity to $83 \%$ (55). However, the inflammation induced by stent placement can lead to abnormal features which can interfere with pCLE assessment (52). A recent meta-analysis of twelve studies involving 591 patients showed that the best diagnostic performance for undetermined pancreaticobiliary strictures is achieved combining CLE and conventional tissue sampling with a sensitivity and specificity of $93 \%$ and $82 \%$, respectively, as compared with $87 \%$ and $76 \%$, respectively, for CLE alone and 64\% and 94\%, respectively, for tissue sampling only (56). The Asia-Pacific consensus recommendations on endoscopic tissue acquisition for biliary strictures state that despite certain limitations, virtual histology by pCLE may be complementary to conventional tissue sampling techniques (57).

Although the data on the accuracy of CLE is promising, a high level of expertise required to interpret CLE in realtime and standardised training is required to improve the interobserver agreement and ensure reproducibility of the published results outside the expert centres. While it 

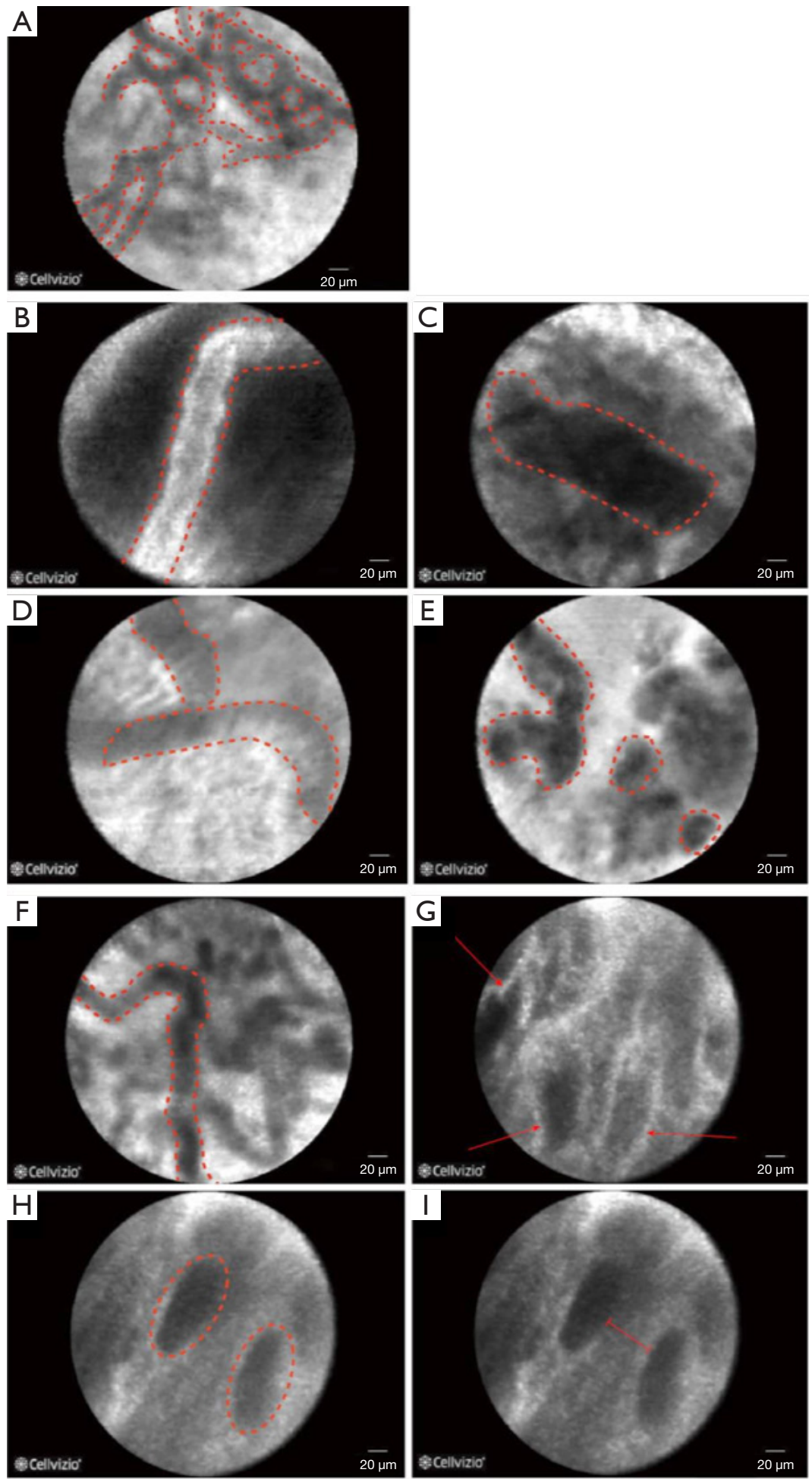

Figure 10 CLE images of biliary ducts. (A) Normal bile duct-reticular network of thin dark branching bands $\leq 20 \mu \mathrm{m}$. (B-E) Features of malignant bile duct strictures: (B) thick white bands $(>20 \mu \mathrm{m}),(\mathrm{C})$ thick dark bands $(>40 \mu \mathrm{m})$, (D) epithelium, (E) dark clumps. (F-J) features of inflammatory bile duct strictures on confocal laser endomicroscopy: (F) thickened reticular structures, $(\mathrm{G})$ multiple white bands, $(\mathrm{H})$ dark granular pattern in scales, (I) increased spaces between scales. Magnification: 1,000x. Adopted from Almadi et al., World 7 Gastroenterol, 2015. CLE, confocal laser endomicroscopy. 
has been shown in one study that inexperienced observers achieved an accuracy of $83 \%$ after three weeks of training by experts, there is no data on the learning curve as operator (58). A study among 6 experienced endoscopists from 5 institutions found that interobserver agreement for individual Miami criteria ranged from poor to fair (59).

Cholangioscopy-guided direct tissue biopsy represents an alternative to CLE in cases where conventional imaging does not achieve conclusive results. There are no studies comparing the diagnostic accuracy of the two techniques.

Finally, there is only one small study that specifically assessed pCLE for the characterization of pancreatic strictures (60), therefore more research is required in this field.

\section{Colon}

Due to a narrow field of view, CLE is impractical for screening, surveillance or improving adenoma detection rate in the colon. Thus, endomicroscopic examination with CLE in colon relies on flagging techniques to target optical biopsies. The Miami classification provided criteria which enable to differentiate neoplastic from non-neoplastic mucosa and characterise colorectal lesions (61). In several studies, these criteria have shown a high sensitivity ranging from $86 \%$ to $100 \%$, specificity of $76 \%$ to $85 \%$, PPV of $55 \%$ to $91 \%$ and NPV of $88 \%$ to $100 \%$ (62-64). Recently, the characteristic features of sessile serrated adenoma/polyps (SSA/Ps) were identified based on a prospective evaluation of 260 CLE images of SSA/Ps from 7 patients. Features of SSA/Ps included a mucus cap with a bright, cloud-like appearance; thin, branching crypts; increased number of goblet cells with dystrophic appearance; microvesicular mucin-containing cells; and architectural disarray (65). These findings require further validation.

During colonoscopy, high diagnostic efficiency is required to allow immediate diagnosis and discard/resect decision-making. Studies have compared CLE with other, already widely available, advanced imaging methods, such as NBI, BLI or iSCAN. A meta-analysis of 102 studies on image-enhanced techniques for optical diagnosis of colorectal lesion showed that the sensitivity and specificity of NBI, i-scan, FICE, and CLE for differentiation between neoplastic and non-neoplastic colonic lesions were high and did not differ significantly (66). Additionally, a study of 65 patients with 130 polyps smaller than $10 \mathrm{~mm}$ comparing prediction of histology by pCLE versus NBI, provided inconclusive results, since pCLE demonstrated higher sensitivity but lower specificity compared with NBI (67).

One Japanese study suggested that pCLE could be used for the prediction of the depth of invasion. Features of early colorectal cancer related to $\mathrm{SM}_{2}$ or deeper invasion based on the Sano's classification, showed that the loss of crypt structure identified had sensitivity, specificity and accuracy of $80 \%, 94 \%, 91 \%$, respectively. The performance of NBI was slightly lower with sensitivity, specificity and accuracy $60 \%, 94 \%, 86 \%$, respectively (68). In summary, the results for CLE in detection and characterization of colorectal neoplasia remain inconclusive and do not support adoption of CLE in the everyday practice. The CLE appearance normal and pathological colonic mucosa are represented in Figure 11.

\section{Inflammatory bowel diseases (IBD)}

More promising is the evidence on the use of endomicroscopy in the evaluation of IBD. Imaging of intestinal inflammation by CLE could be used to diagnose IBD, differentiate Crohn's disease (CD) from ulcerative colitis (UC), assess the severity of inflammation, predict relapse or therapeutic response and detect dysplasia (69-71). The characteristic features of inflamed bowel mucosa on CLE examination include irregular and tortuous crypts with wider lumens, an increased density of epithelial gaps and fluorescein leakage to the interstitial space (72-75) (Figure 12). Several studies show that CLE provides reliable assessment of the degree of inflammation and disease activity in patients with IBD with high correlation with the inflammatory changes compared to conventional histopathology (76-79).

Perhaps, even more interestingly, CLE enables to identify IBD-associated changes in macroscopically non-inflamed mucosa. Inflammatory activity is classified using a fourgrade system combining morphological irregularity of the crypts and microvascular architecture with fluorescein leak. Based on these criteria, it was found that CLE could predict UC activity more accurately than WLE as more than half of patients with normal mucosa seen on conventional WLE showed acute inflammation on histology, while normal CLE findings were highly predictive of histological remission (77). Thus, CLE might be particularly useful in the emerging "treat-to-target" approach in IBD, which involves achieving mucosal healing in patients with IBD $(71,80)$. CLE has also been evaluated as a prognostic indicator of IBD relapse by assessing defects in intestinal barrier function of epithelial cells and the tight junction (79). CLE evidence 

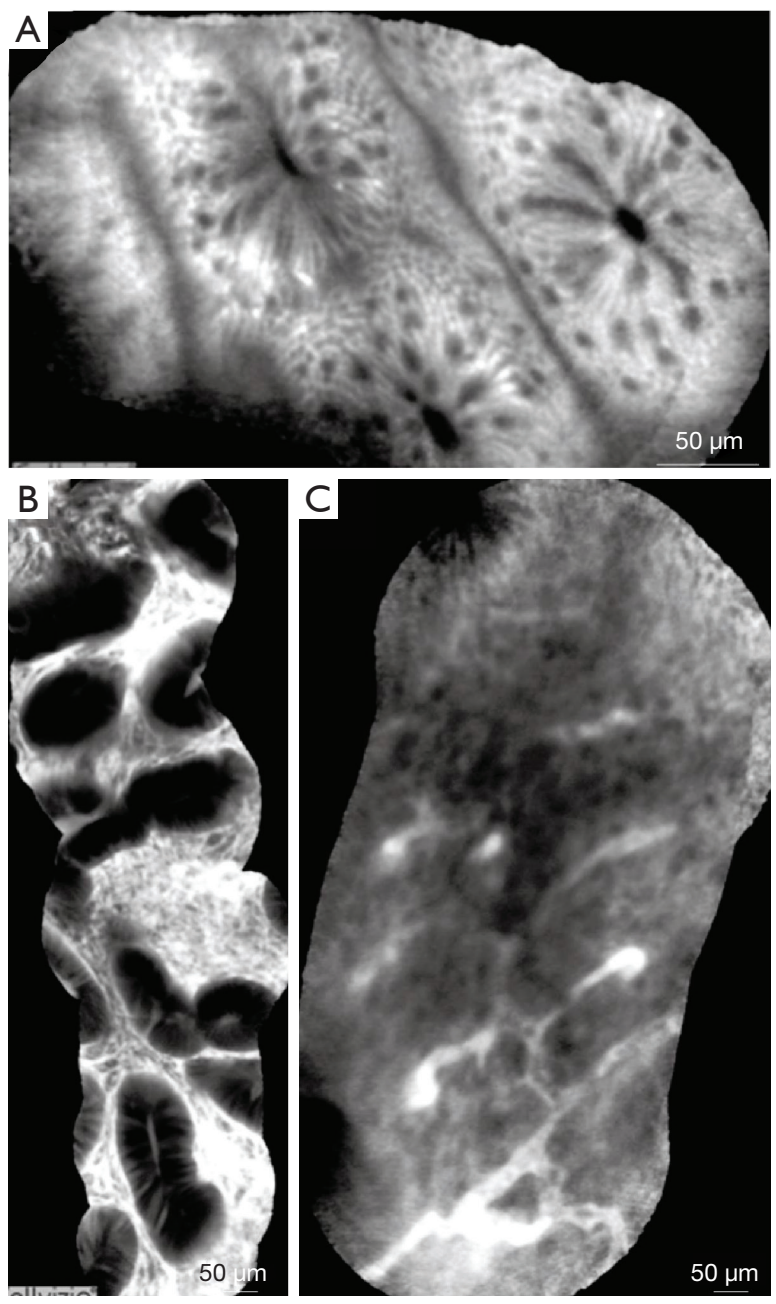

Figure 11 CLE images of colonic mucosa. (A) Normal colonic mucosa-round shaped crypts, dark goblet cells, narrow and regular blood vessels surrounding the crypts. (B) Adenomatous polyp-irregular or villiform structures and a dark, irregularly thickened epithelium with a decreased number of goblet cells. (C) Adenocarcinoma-disorganized mucosa, lack of structure, elongated crypts, irregularly thickened epithelium, dilated and distorted blood vessels. Adapted from De Palma GD, et al., World f Gastroenterol, 2009. CLE, confocal laser endomicroscopy.

of increased cell shedding with fluorescein leakage appears to precede relapse within 12 months in patients in clinical remission. The sensitivity, specificity, and accuracy of this feature to predict future flare ups were $62 \%, 91 \%$ and $79 \%$, respectively (79).

Although CLE enables to visualise several disease-specific microscopic features used in standard histopathology, which may help to distinguish between $\mathrm{UC}$ and $\mathrm{CD}$, due to a limited penetration depth of CLE, subtle submucosal details and granulomas cannot be evaluated. Nevertheless, recently described CLE-IBD differentiation score, based on endomicroscopy assessment revealed accuracy of $93.7 \%$ when compared with the historical clinical diagnosis and the histopathological gold standard. CLE findings in patients with Crohn's disease, showed significantly more discontinuous inflammation ( $87.5 \%$ vs. $5.1 \%$ ), focal cryptitis (75.0\% vs. $12.8 \%)$, and discontinuous crypt architectural abnormality $(87.5 \%$ vs. $10.3 \%)$ than in ulcerative colitis $(\mathrm{P}<0.0001)$. Conversely, ulcerative colitis was associated with severe, widespread crypt distortion $(87.2 \%$ vs. $17.5 \%$ in Crohn's disease), decreased crypt density $(79.5 \%$ vs. $22.5 \%)$, and irregular surface $(89.7 \%$ vs. $17.5 \% ; \mathrm{P}<0.0001$ for all comparisons) (81).

Several studies have examined the potential role of 

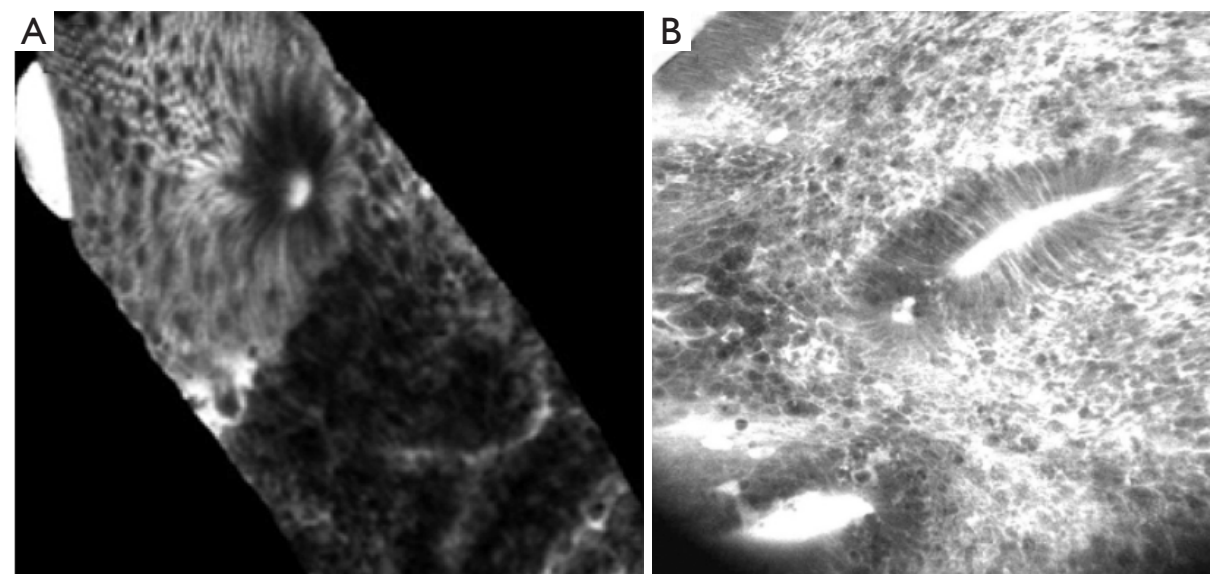

Figure 12 CLE images in inflammatory bowel diseases. (A) Active ulcerative colitis (distal colitis-the switch from normal mucosa (top of the figure) to inflamed mucosa with irregular arrangement of crypts, crypt fusion and capillaries alterations. (B) Crohn's disease-dilated and distorted capillaries, altered crypt architecture, increased distance between the crypts. Magnification: 1,000x. Adapted from De Palma GD, et al., World $\mathcal{F}$ Gastroenterol, 2013. CLE, confocal laser endomicroscopy.

CLE in the surveillance of patients with IBD (82-85). The CLE images indicative for dysplasia include ridgedlined irregular epithelial layer of cells, loss of crypts and goblet cells, distorted and dilated vessels with increased leakage (83). A meta-analysis of fifteen studies involving 719 patients showed that the pooled sensitivity and specificity for diagnosing colorectal neoplasia in IBD patients with CLE was $94 \%$ and $95 \%$, respectively (85). Moreover, eCLE combined with chromoendoscopy (CE) showed a nearly 5 -fold increase in the detection of neoplasia, with $50 \%$ fewer biopsies compared with WLE and chromoendoscopy, with overall sensitivity and specificity for neoplasia detection of $95 \%$ and $98 \%$, respectively (82). CE-guided pCLE has been used to predict dysplasia with a sensitivity of $100 \%$, specificity of $88.5 \%$, PPV of $91.2 \%$, and NPV of $100 \%$, while chromoendoscopy alone showed sensitivity of $96.8 \%$, specificity of $69.2 \%$, PPV of $78.9 \%$, and NPV of $94.7 \%$ (83). Despite encouraging level of diagnostic accuracy, the advantage of pCLE in diagnosing IBD-associated dysplasia is yet to be established mainly due to the narrow field of view, which restricts ability to perform pan-colonic examination and the time limitation of combining CLE with chromoendoscopy.

Finally, two case series suggested that CLE appears to be able to diagnose collagenous colitis as CLE permits to localize and measure the number of collagenous bands in the mucosal layer. Patients with collagenous colitis showed a well-defined "shell" around the crypts at a depth between 15 and $70 \mathrm{~mm}$ of the wall corresponding to the patchy increase in the thickness of the subepithelial collagenous plate described on histopathology $(86,87)$.

In summary, advances in CLE imaging in IBD patients may be used not only to better understand the pathophysiology of the disease but also to guide optimized therapy and thus allow a completely new, personalized approach to IBD management. Currently, CLE is utilized primarily as an additional diagnostic tool in patients with IBD only in tertiary centres and, so far, it has not been included in any official guidelines on IBD management.

\section{Future directions}

In addition to provide insight into the morphology of GI epithelia, CLE is well placed to enable visualization of cellular function and characterization of molecular processes in vivo in combination with molecular probes $(88,89)$. For example, a recent study with topical administration of fluorescent anti-TNF (adalimumab) in 25 patients with $\mathrm{CD}$, detected intestinal membrane-bound TNF-mTNF1 immune cells. The quantitative image analysis revealed that a high amount of mTNF was associated with substantially higher short-term clinical response rates (92\%) at week 12 as compared with the patients with a low level of mTNF $(15 \%)$ (90). Targeted, peptide-based imaging agents are also promising for future use in early detection of cancer. Recently, a fluorescent-labelled monoclonal antibody against $M G 7-A g$, a novel tumor-associated antigen which is expressed in $94 \%$ of gastric cancers and $83.6 \%$ of sera from patients with gastric carcinoma, was used for in vivo 
imaging by CLE in xenografts and ex vivo imaging in human resection specimens. The staining was positive in $96 \%$ of gastric cancer specimens and $22 \%$ of non-neoplastic tissues (91). Similarly, the vascular homing peptide GEBP11 was evaluated as a target for the molecular imaging of gastric cancer in human tissue. A specific signal was observed in $93 \%$ of gastric cancer specimens but also in $29 \%$ of nonneoplastic specimens (92). Moreover, two animal in vivo studies have shown the feasibility of assessing epidermal growth factor receptor (EGFR) expression patterns by pCLE with labelled antibodies against the EGFR receptor $(93,94)$. Finally, in the context of BE, a FITC labeled peptide (ASY) with high affinity for esophageal cancer cell lines was used as topical spray in 25 patients and could be imaged in vivo by pCLE. Based on the level fluorescence intensity this molecular imaging approach showed a sensitivity and specificity of $75 \%$ and $97 \%$, respectively, for a diagnosis of early BE-related neoplasia (95). Altogether, these data suggest a potential role of CLE-based molecular imaging in the endoscopic diagnosis of cancer and in the evaluation of response to targeted anti-cancer and anti-inflammatory therapies.

Although most of the data published so far refer to luminal pathology, the possibility to reach with nCLE parenchymal organs also opens new avenues in the real-time optical biopsies of solid lesions. In this direction, a future avenue of research could be the combination of intratumoral injection of fluorescent labels and nCLE imaging of regional lymph nodes to refine the sentinel lymph node technique.

The advent of artificial intelligence in medical diagnostic might complement well with CLE diagnosis. Although data on training show quick learning curve even by non-experienced operators, most of the datasets for training are based on good quality images, which are often challenging to generate in clinical practice. Computer assisted diagnosis has the potential to help the operator interpret images in real time during CLE procedures and represent an interesting area of future investigation.

In summary, although CLE provides detailed and highresolution insight into the morphology of GI epithelia, the narrow field of view remains the main limitation. Therefore, in the future it will be vital to combine CLE with appropriate red-flag techniques in order to limit the sampling error. Molecular probes can take CLE to a next level of performance. In the conventional diagnostic applications, multi-center, randomized studies will be required for definitive evidence. Finally, more rigorous standardisation of operator training is required to ensure reproducibility of results.

\section{Acknowledgments}

Funding: None.

\section{Footnote}

Provenance and Peer Review: This article was commissioned by the Guest Editors (Krish Ragunath, Philip W. Y. Chiu) for the series "Advanced Endoscopic Imaging of the GI Tract" published in Translational Gastroenterology and Hepatology. The article has undergone external peer review.

Conflicts of Interest: All authors have completed the ICMJE uniform disclosure form (available at https://tgh.amegroups. com/article/view/10.21037/tgh.2020.04.02/coif). The series "Advanced endoscopic imaging of the GI tract" was commissioned by the editorial office without any funding or sponsorship. The authors have no other conflicts of interest to declare.

Ethical Statement: The authors are accountable for all aspects of the work in ensuring that questions related to the accuracy or integrity of any part of the work are appropriately investigated and resolved.

Open Access Statement: This is an Open Access article distributed in accordance with the Creative Commons Attribution-NonCommercial-NoDerivs 4.0 International License (CC BY-NC-ND 4.0), which permits the noncommercial replication and distribution of the article with the strict proviso that no changes or edits are made and the original work is properly cited (including links to both the formal publication through the relevant DOI and the license). See: https://creativecommons.org/licenses/by-nc-nd/4.0/.

\section{References}

1. Goetz M, Toermer T, Vieth M, et al. Simultaneous confocal laser endomicroscopy and chromoendoscopy with topical cresyl violet. Gastrointest Endosc 2009;70:959-68.

2. Li CQ, Yu T, Zuo XL, et al. Effects on confocal laser endomicroscopy image quality by different acriflavine concentrations. J Interv Gastroenterol 2011;1:59-63.

3. Meyer RR, Simpson MV. DNA biosynthesis in mitochondria. Differential inhibition of mitochondrial and 
nuclear DNA polymerases by the mutagenic dyes ethidium bromide and acriflavin. Biochem Biophys Res Commun 1969;34:238-44.

4. Caulfield MJ, Burleson GR, Pollard M. Ozonation of mutagenic and carcinogenic alkylating agents, pesticides, aflatoxin B1, and benzidine in water. Cancer Res 1979;39:2155-9.

5. Wallace MB, Meining A, Canto MI, et al. The safety of intravenous fluorescein for confocal laser endomicroscopy in the gastrointestinal tract. Aliment Pharmacol Ther 2010;31:548-52.

6. Rzouq F, Vennalaganti P, Pakseresht K, et al. In-class didactic versus self-directed teaching of the probebased confocal laser endomicroscopy (pCLE) criteria for Barrett's esophagus. Endoscopy 2016;48:123-7.

7. Pittayanon R, Rerknimitr R, Wisedopas N, et al. The learning curve of gastric intestinal metaplasia interpretation on the images obtained by probe-based confocal laser endomicroscopy. Diagn Ther Endosc 2012;2012:278045.

8. Kuiper T, Kiesslich R, Ponsioen C, et al. The learning curve, accuracy, and interobserver agreement of endoscope-based confocal laser endomicroscopy for the differentiation of colorectal lesions. Gastrointest Endosc 2012;75:1211-7.

9. Gaddam S, Mathur SC, Singh M, et al. Novel probe-based confocal laser endomicroscopy criteria and interobserver agreement for the detection of dysplasia in Barrett's esophagus. Am J Gastroenterol 2011;106:1961-9.

10. Buchner AM, Gomez V, Heckman MG, et al. The learning curve of in vivo probe-based confocal laser endomicroscopy for prediction of colorectal neoplasia. Gastrointest Endosc 2011;73:556-60.

11. Fitzgerald RC, di Pietro M, Ragunath K, et al. British Society of Gastroenterology guidelines on the diagnosis and management of Barrett's oesophagus. Gut 2014;63:7-42.

12. Shaheen NJ, Falk GW, Iyer PG, et al. Corrigendum: ACG Clinical Guideline: Diagnosis and Management of Barrett's Esophagus. Am J Gastroenterol 2016;111:1077.

13. Neumann H, Langner C, Neurath MF, et al. Confocal Laser Endomicroscopy for Diagnosis of Barrett's Esophagus. Front Oncol 2012;2:42.

14. di Pietro M, Bertani H, O’Donovan M, et al. Development and Validation of Confocal Endomicroscopy Diagnostic Criteria for Low-Grade Dysplasia in Barrett's Esophagus. Clin Transl Gastroenterol 2019;10:e00014.

15. Sharma P, Meining AR, Coron E, et al. Real-time increased detection of neoplastic tissue in Barrett's esophagus with probe-based confocal laser endomicroscopy: final results of an international multicenter, prospective, randomized, controlled trial. Gastrointest Endosc 2011;74:465-72.

16. Canto MI, Anandasabapathy S, Brugge W, et al. In vivo endomicroscopy improves detection of Barrett's esophagus-related neoplasia: a multicenter international randomized controlled trial (with video). Gastrointest Endosc 2014;79:211-21.

17. Wu J, Pan YM, Wang TT, et al. Confocal laser endomicroscopy for detection of neoplasia in Barrett's esophagus: a meta-analysis. Dis Esophagus 2014;27:248-54.

18. di Pietro M, Bird-Lieberman EL, Liu X, et al. Autofluorescence-Directed Confocal Endomicroscopy in Combination With a Three-Biomarker Panel Can Inform Management Decisions in Barrett's Esophagus. Am J Gastroenterol 2015;110:1549-58.

19. Pech O, Rabenstein T, Manner H, et al. Confocal laser endomicroscopy for in vivo diagnosis of early squamous cell carcinoma in the esophagus. Clin Gastroenterol Hepatol 2008;6:89-94.

20. Safatle-Ribeiro AV, Baba ER, Faraj SF, et al. Diagnostic accuracy of probe-based confocal laser endomicroscopy in Lugol-unstained esophageal superficial lesions of patients with head and neck cancer. Gastrointest Endosc 2017;85:1195-207.

21. Zhang JN, Li YQ, Zhao YA, et al. Classification of gastric pit patterns by confocal endomicroscopy. Gastrointest Endosc 2008;67:843-53.

22. Li Z, Zuo XL, Li CQ, et al. New Classification of Gastric Pit Patterns and Vessel Architecture Using Probe-based Confocal Laser Endomicroscopy. J Clin Gastroenterol 2016;50:23-32.

23. He XK, Liu D, Sun LM. Diagnostic performance of confocal laser endomicroscopy for optical diagnosis of gastric intestinal metaplasia: a meta-analysis. BMC Gastroenterol 2016;16:109.

24. Qian W, Bai T, Wang H, et al. Meta-analysis of confocal laser endomicroscopy for the diagnosis of gastric neoplasia and adenocarcinoma. J Dig Dis 2016;17:366-76.

25. Li Z, Zuo XL, Yu T, et al. Confocal laser endomicroscopy for in vivo detection of gastric intestinal metaplasia: a randomized controlled trial. Endoscopy 2014;46:282-90.

26. Pittayanon R, Rerknimitr R, Wisedopas N, et al. Flexible spectral imaging color enhancement plus probe-based confocal laser endomicroscopy for gastric intestinal metaplasia detection. J Gastroenterol Hepatol 2013;28:1004-9.

27. Zuo XL, Li Z, Li CQ, et al. Probe-based endomicroscopy 
for in vivo detection of gastric intestinal metaplasia and neoplasia: a multicenter randomized controlled trial. Endoscopy 2017;49:1033-42.

28. Lim LG, Yeoh KG, Srivastava S, et al. Comparison of probe-based confocal endomicroscopy with virtual chromoendoscopy and white-light endoscopy for diagnosis of gastric intestinal metaplasia. Surg Endosc 2013;27:4649-55.

29. Kiesslich R, Goetz M, Burg J, et al. Diagnosing Helicobacter pylori in vivo by confocal laser endoscopy. Gastroenterology 2005;128:2119-23.

30. Fugazza A, Gaiani F, Carra MC, et al. Confocal Laser Endomicroscopy in Gastrointestinal and Pancreatobiliary Diseases: A Systematic Review and Meta-Analysis. Biomed Res Int 2016;2016:4638683.

31. Ohmiya N, Horiguchi N, Tahara T, et al. In vivo characterization of abnormalities in small-bowel diseases using probe-based confocal laser endomicroscopy. Endosc Int Open 2017;5:E547-58.

32. Trovato C, Sonzogni A, Ravizza D, et al. Celiac disease: in vivo diagnosis by confocal endomicroscopy. Gastrointest Endosc 2007;65:1096-9.

33. Günther U, Daum S, Heller F, et al. Diagnostic value of confocal endomicroscopy in celiac disease. Endoscopy 2010;42:197-202.

34. Leong RW, Nguyen NQ, Meredith CG, et al. In vivo confocal endomicroscopy in the diagnosis and evaluation of celiac disease. Gastroenterology 2008;135:1870-6.

35. Pittayanon R, Rerknimitr R, Imraporn B, et al. Diagnostic values of dual focus narrow band imaging and probe-based confocal laser endomicroscopy in FAP-related duodenal adenoma. Endosc Int Open 2015;3:E450-5.

36. Fritscher-Ravens A, Schuppan D, Ellrichmann M, et al. Confocal endomicroscopy shows food-associated changes in the intestinal mucosa of patients with irritable bowel syndrome. Gastroenterology 2014;147:1012-20.e4.

37. Modi RM, Swanson B, Muscarella P, 2nd, et al. Novel techniques for diagnosis of serous cystadenoma: fern pattern of vascularity confirmed by in vivo and ex vivo confocal laser endomicroscopy. Gastrointest Endosc 2017;85:258-9.

38. Napoleon B, Lemaistre AI, Pujol B, et al. In vivo characterization of pancreatic cystic lesions by needlebased confocal laser endomicroscopy (nCLE): proposition of a comprehensive nCLE classification confirmed by an external retrospective evaluation. Surg Endosc 2016;30:2603-12.

39. Krishna SG, Brugge WR, Dewitt JM, et al. Needlebased confocal laser endomicroscopy for the diagnosis of pancreatic cystic lesions: an international external interobserver and intraobserver study (with videos). Gastrointest Endosc 2017;86:644-54.e2.

40. Nakai Y, Iwashita T, Park DH, et al. Diagnosis of pancreatic cysts: EUS-guided, through-the-needle confocal laserinduced endomicroscopy and cystoscopy trial: DETECT study. Gastrointest Endosc 2015;81:1204-14.

41. Krishna SG, Swanson B, Hart PA, et al. Validation of diagnostic characteristics of needle based confocal laser endomicroscopy in differentiation of pancreatic cystic lesions. Endosc Int Open 2016;4:E1124-35.

42. Napoleon B, Palazzo M, Lemaistre AI, et al. Needle-based confocal laser endomicroscopy of pancreatic cystic lesions: a prospective multicenter validation study in patients with definite diagnosis. Endoscopy 2019;51:825-35.

43. Teubner D, Kiesslich R, Matsumoto T, et al. Beyond standard image-enhanced endoscopy confocal endomicroscopy. Gastrointest Endosc Clin N Am 2014;24:427-34.

44. Konda VJ, Meining A, Jamil LH, et al. A pilot study of in vivo identification of pancreatic cystic neoplasms with needle-based confocal laser endomicroscopy under endosonographic guidance. Endoscopy 2013;45:1006-13.

45. Kadayifci A, Atar M, Basar O, et al. Needle-Based Confocal Laser Endomicroscopy for Evaluation of Cystic Neoplasms of the Pancreas. Dig Dis Sci 2017;62:1346-53.

46. Giovannini M, Caillol F, Monges G, et al. Endoscopic ultrasound-guided needle-based confocal laser endomicroscopy in solid pancreatic masses. Endoscopy 2016;48:892-8.

47. Kongkam P, Pittayanon R, Sampatanukul P, et al. Endoscopic ultrasound-guided needle-based confocal laser endomicroscopy for diagnosis of solid pancreatic lesions (ENES): a pilot study. Endosc Int Open 2016;4:E17-23.

48. Karstensen JG, Cartana T, Constantinescu C, et al. Endoscopic ultrasound guided needle-based confocal laser endomicroscopy in solid pancreatic masses - a prospective validation study. Endosc Int Open 2018;6:E78-85.

49. Ponchon T, Gagnon P, Berger F, et al. Value of endobiliary brush cytology and biopsies for the diagnosis of malignant bile duct stenosis: results of a prospective study. Gastrointest Endosc 1995;42:565-72.

50. Navaneethan U, Njei B, Lourdusamy V, et al. Comparative effectiveness of biliary brush cytology and intraductal biopsy for detection of malignant biliary strictures: a systematic review and meta-analysis. Gastrointest Endosc 2015;81:168-76.

51. Slivka A, Gan I, Jamidar P, et al. Validation of the 
diagnostic accuracy of probe-based confocal laser endomicroscopy for the characterization of indeterminate biliary strictures: results of a prospective multicenter international study. Gastrointest Endosc 2015;81:282-90.

52. Meining A, Chen YK, Pleskow D, et al. Direct visualization of indeterminate pancreaticobiliary strictures with probe-based confocal laser endomicroscopy: a multicenter experience. Gastrointest Endosc 2011;74:961-8.

53. Caillol F, Bories E, Autret A, et al. Evaluation of pCLE in the bile duct: final results of EMID study: pCLE: impact in the management of bile duct strictures. Surg Endosc 2015;29:2661-8.

54. Meining A, Shah RJ, Slivka A, et al. Classification of probe-based confocal laser endomicroscopy findings in pancreaticobiliary strictures. Endoscopy 2012;44:251-7.

55. Caillol F, Filoche B, Gaidhane M, et al. Refined probebased confocal laser endomicroscopy classification for biliary strictures: the Paris Classification. Dig Dis Sci 2013;58:1784-9.

56. Gao YD, Qu YW, Liu HF. Comparison of diagnostic efficacy between CLE, tissue sampling, and CLE combined with tissue sampling for undetermined pancreaticobiliary strictures: a meta-analysis. Scand J Gastroenterol 2018;53:482-9.

57. Sun B, Moon JH, Cai Q, et al. Review article: AsiaPacific consensus recommendations on endoscopic tissue acquisition for biliary strictures. Aliment Pharmacol Ther 2018;48:138-51.

58. Anderson MA, Appalaneni V, Ben-Menachem T, et al. The role of endoscopy in the evaluation and treatment of patients with biliary neoplasia. Gastrointest Endosc 2013;77:167-74.

59. Talreja JP, Turner BG, Gress FG, et al. Pre- and posttraining session evaluation for interobserver agreement and diagnostic accuracy of probe-based confocal laser endomicroscopy for biliary strictures. Dig Endosc 2014;26:577-80.

60. Kahaleh M, Turner BG, Bezak K, et al. Probe-based confocal laser endomicroscopy in the pancreatic duct provides direct visualization of ductal structures and aids in clinical management. Dig Liver Dis 2015;47:202-4.

61. Wallace M, Lauwers GY, Chen Y, et al. Miami classification for probe-based confocal laser endomicroscopy. Endoscopy 2011;43:882-91.

62. De Palma GD, Staibano S, Siciliano S, et al. In vivo characterisation of superficial colorectal neoplastic lesions with high-resolution probe-based confocal laser endomicroscopy in combination with video-mosaicing: a feasibility study to enhance routine endoscopy. Dig Liver Dis 2010;42:791-7.

63. Shahid MW, Buchner AM, Coron E, et al. Diagnostic accuracy of probe-based confocal laser endomicroscopy in detecting residual colorectal neoplasia after EMR: a prospective study. Gastrointest Endosc 2012;75:525-33.

64. Buchner AM, Shahid MW, Heckman MG, et al. Comparison of probe-based confocal laser endomicroscopy with virtual chromoendoscopy for classification of colon polyps. Gastroenterology 2010;138:834-42.

65. Parikh ND, Gibson J, Nagar A, et al. Confocal laser endomicroscopy features of sessile serrated adenomas/ polyps. United European Gastroenterol J 2016;4:599-603.

66. Wanders LK, East JE, Uitentuis SE, et al. Diagnostic performance of narrowed spectrum endoscopy, autofluorescence imaging, and confocal laser endomicroscopy for optical diagnosis of colonic polyps: a meta-analysis. Lancet Oncol 2013;14:1337-47.

67. Shahid MW, Buchner AM, Heckman MG, et al. Diagnostic accuracy of probe-based confocal laser endomicroscopy and narrow band imaging for small colorectal polyps: a feasibility study. Am J Gastroenterol 2012;107:231-9.

68. Abe S, Saito Y, Oono Y, et al. Pilot study on probe-based confocal laser endomicroscopy for colorectal neoplasms: an initial experience in Japan. Int J Colorectal Dis 2018;33:1071-8.

69. Rasmussen DN, Karstensen JG, Riis LB, et al. Confocal Laser Endomicroscopy in Inflammatory Bowel Disease--A Systematic Review. J Crohns Colitis 2015;9:1152-9.

70. Hundorfean G, Chiriac MT, Mudter J, et al. Confocal laser endomicroscopy provides potential differentiation criteria between Crohn's disease and ulcerative colitis. Inflamm Bowel Dis 2013;19:E61-4.

71. Neurath MF, Travis SP. Mucosal healing in inflammatory bowel diseases: a systematic review. Gut 2012;61:1619-35.

72. Musquer N, Coquenlorge S, Bourreille A, et al. Probebased confocal laser endomicroscopy: a new method for quantitative analysis of pit structure in healthy and Crohn's disease patients. Dig Liver Dis 2013;45:487-92.

73. Lim LG, Neumann J, Hansen T, et al. Confocal endomicroscopy identifies loss of local barrier function in the duodenum of patients with Crohn's disease and ulcerative colitis. Inflamm Bowel Dis 2014;20:892-900.

74. Liu JJ, Madsen KL, Boulanger P, et al. Mind the gaps: confocal endomicroscopy showed increased density of small bowel epithelial gaps in inflammatory bowel disease. 
J Clin Gastroenterol 2011;45:240-5.

75. Liu JJ, Wong K, Thiesen AL, et al. Increased epithelial gaps in the small intestines of patients with inflammatory bowel disease: density matters. Gastrointest Endosc 2011;73:1174-80.

76. Neumann H, Vieth $M$, Gunther C, et al. Virtual chromoendoscopy for prediction of severity and disease extent in patients with inflammatory bowel disease: a randomized controlled study. Inflamm Bowel Dis 2013;19:1935-42.

77. Li CQ, Xie XJ, Yu T, et al. Classification of inflammation activity in ulcerative colitis by confocal laser endomicroscopy. Am J Gastroenterol 2010;105:1391-6.

78. Buda A, Hatem G, Neumann H, et al. Confocal laser endomicroscopy for prediction of disease relapse in ulcerative colitis: a pilot study. J Crohns Colitis 2014;8:304-11.

79. Kiesslich R, Duckworth CA, Moussata D, et al. Local barrier dysfunction identified by confocal laser endomicroscopy predicts relapse in inflammatory bowel disease. Gut 2012;61:1146-53.

80. Neurath MF. New targets for mucosal healing and therapy in inflammatory bowel diseases. Mucosal Immunol 2014;7:6-19.

81. Tontini GE, Mudter J, Vieth M, et al. Confocal laser endomicroscopy for the differential diagnosis of ulcerative colitis and Crohn's disease: a pilot study. Endoscopy 2015;47:437-43.

82. Kiesslich R, Goetz M, Lammersdorf K, et al. Chromoscopy-guided endomicroscopy increases the diagnostic yield of intraepithelial neoplasia in ulcerative colitis. Gastroenterology 2007;132:874-82.

83. Hlavaty T, Huorka M, Koller T, et al. Colorectal cancer screening in patients with ulcerative and Crohn's colitis with use of colonoscopy, chromoendoscopy and confocal endomicroscopy. Eur J Gastroenterol Hepatol 2011;23:680-9.

84. van den Broek FJ, van Es JA, van Eeden S, et al. Pilot study of probe-based confocal laser endomicroscopy during colonoscopic surveillance of patients with longstanding ulcerative colitis. Endoscopy 2011;43:116-22.

doi: $10.21037 / \operatorname{tgh} .2020 .04 .02$

Cite this article as: Pilonis ND, Januszewicz W, di Pietro M. Confocal laser endomicroscopy in gastro-intestinal endoscopy: technical aspects and clinical applications. Transl Gastroenterol Hepatol 2022;7:7.
85. Su P, Liu Y, Lin S, et al. Efficacy of confocal laser endomicroscopy for discriminating colorectal neoplasms from non-neoplasms: a systematic review and metaanalysis. Colorectal Dis 2013;15:e1-12.

86. Zambelli A, Villanacci V, Buscarini E, et al. Collagenous colitis: a case series with confocal laser microscopy and histology correlation. Endoscopy 2008;40:606-8.

87. Kiesslich R, Hoffman A, Goetz M, et al. In vivo diagnosis of collagenous colitis by confocal endomicroscopy. Gut 2006;55:591-2.

88. De Palma GD, Colavita I, Zambrano G, et al. Detection of colonic dysplasia in patients with ulcerative colitis using a targeted fluorescent peptide and confocal laser endomicroscopy: A pilot study. PLoS One 2017;12:e180509.

89. Hsiung PL, Hardy J, Friedland S, et al. Detection of colonic dysplasia in vivo using a targeted heptapeptide and confocal microendoscopy. Nat Med 2008;14:454-8.

90. Atreya $\mathrm{R}$, Neumann $\mathrm{H}$, Neufert C, et al. In vivo imaging using fluorescent antibodies to tumor necrosis factor predicts therapeutic response in Crohn's disease. Nat Med 2014;20:313-8.

91. Li Z, Zuo XL, Li CQ, et al. In vivo molecular imaging of gastric cancer by targeting MG7 antigen with confocal laser endomicroscopy. Endoscopy 2013;45:79-85.

92. Liu L, Yin J, Liu C, et al. In vivo molecular imaging of gastric cancer in human-murine xenograft models with confocal laser endomicroscopy using a tumor vascular homing peptide. Cancer Lett 2015;356:891-8.

93. Nakai Y, Shinoura S, Ahluwalia A, et al. Molecular imaging of epidermal growth factor-receptor and survivin in vivo in porcine esophageal and gastric mucosae using probe-based confocal laser-induced endomicroscopy: proof of concept. J Physiol Pharmacol 2012;63:303-7.

94. Hoetker MS, Kiesslich R, Diken M, et al. Molecular in vivo imaging of gastric cancer in a human-murine xenograft model: targeting epidermal growth factor receptor. Gastrointest Endosc 2012;76:612-20.

95. Sturm MB, Joshi BP, Lu S, et al. Targeted imaging of esophageal neoplasia with a fluorescently labeled peptide: first-in-human results. Sci Transl Med 2013;5:184ra61. 